\title{
Convergence of the Godunov Scheme for a Simplified One-Dimensional Hydrodynamic Model for Semiconductor Devices
}

\author{
Bo Zhang \\ Department of Mathematics, Purdue University, West Lafayette, IN 47907, USA
}

Received May 22, 1992; in revised form February 1, 1993

\begin{abstract}
Convergence of approximate solutions derived by the Godunov scheme for a simplified one-dimensional hydrodynamic model for semiconductor devices is established by using the compensated compactness method. A global existence theorem is shown; and a numerical method for the computation of the physical global solution of this model is provided by this approach.
\end{abstract}

\section{Introduction and Main Result}

The hydrodynamic model for semiconductor devices plays an important role in simulating the behavior of the charge carrier in submicron semiconductor devices since it exhibits velocity overshoot and ballistic effects which are not accounted for in the classical drift-diffusion model $[1,2]$. The hydrodynamic model consists of a set of nonlinear Euler equations for particle density, current density, and energy density. The Poisson equation for the electrostatic potential is also used. We investigate a simplified hydrodynamic model in which pressure is a given function of the particle density only.

After appropriate scaling, the one-dimensional time-dependent system in the case of one carrier type (e.g., electrons) reads [7]:

$$
\begin{aligned}
& \rho_{t}+(\rho u)_{x}=0, \\
& (\rho u)_{t}+\left(\rho u^{2}+p(\rho)\right)_{x}=\rho \phi_{x}-\frac{\rho u}{\tau}, \\
& \phi_{x x}=\rho-D(x),
\end{aligned}
$$

where $\rho(x, t), u(x, t), \phi(x, t)$ denote the electron density, velocity, and the electrostatic potential, respectively. The pressure function, $p=p(\rho)$, has the property that $\rho^{2} p^{\prime}(\rho)$ is strictly monotonically increasing from $[0, \infty)$ onto $[0, \infty)$. A commonly-used hypothesis is [6]:

$$
p(\rho)=k \rho^{\gamma}, \quad \gamma>1, \quad k>0 .
$$


For simplicity, we take $k=\frac{1}{\gamma}$ although the main result of this paper holds for every positive constant $k$. The momentum relaxation time of $\tau=\tau(\rho, \rho u)$ is used, and we assume that

$$
\tau(\rho, \rho u) \geqq \tau_{0}>0, \quad \text { for any }(\rho, \rho u) \in[0, \infty) \times \mathbb{R} .
$$

The device domain is the $x$-interval $I \equiv(0,1)$. The doping profile $D=D(x)$ is assumed to be such that

$$
D(x) \in L^{\infty}(0,1) .
$$

After introducing the current density $m=\rho u$, we can rewrite (1.1)-(1.3) as follows:

$$
\begin{aligned}
& \rho_{t}+m_{x}=0, \\
& m_{t}+\left(\frac{m^{2}}{\rho}+p(\rho)\right)_{x}=\rho \phi_{x}-\frac{m}{\tau}, \\
& \phi_{x x}=\rho-D(x) .
\end{aligned}
$$

The system $(1.1)^{\prime}-(1.3)^{\prime}$ is supplemented by the following initial-boundary value conditions:

$$
\begin{aligned}
& \left.(\rho, m)\right|_{t=0}=\left(\rho_{0}(x), m_{0}(x)\right), \quad 0<x<1, \\
& \left.m\right|_{x=0}=m_{1}(t) \equiv \rho(0, t) u_{1}(t),\left.\quad m\right|_{x=1}=m_{2}(t) \equiv \rho(1, t) u_{2}(t), \quad t \geqq 0, \\
& \left.\phi\right|_{x=0}=\phi_{1}(t),\left.\quad \phi\right|_{x=1}=\phi_{2}(t), \quad t \geqq 0,
\end{aligned}
$$

where $\phi_{i}, i=1,2$, stand for the applied bias. The boundary data $m_{1}(t)$ and $m_{2}(t)$ must be chosen carefully because:

(1) The problem is overdetermined if one gives the data on both $\rho$ and $u$ at $x=0$ or $x=1$.

(2) The problem is not well-posed if one does not provide supplementary conditions on the boundary data.

For example, the density $\rho$ blows up for some boundary data for the double piston problem [24]. For problem $(1.1)^{\prime}-(1.3)^{\prime}$, it is no surprise that if the applied inflow current $m_{1}(t)$ or $m_{2}(t)$ is large enough the solution of $(1.1)^{\prime}-(1.3)^{\prime}$ blows up in finite time. The mathematical analysis of this interesting physical phenomenon will be presented in a forthcoming paper. In this paper, we investigate a special case of boundary data $\left(u_{1}(t)=u_{2}(t)=0\right.$ for all $\left.t \geqq 0\right)$ from the mathematical point of view.

There has recently been some mathematical analysis of the hydrodynamic model (1.1)-(1.3). In [7] the authors proved the existence of a unique smooth solution from the steady-state of (1.1)-(1.3) in the subsonic case, which is characterized by a smallness assumption on the current flowing through the device. The existence of a local smooth solution of the time-dependent problem (1.1)-(1.3) was proved by using Lagrangian mass coordinates [31]. Due to the formation of shocks, we cannot expect to obtain global smooth solution in the general case. For example, the numerical simulation of a steady-state shock wave in the hydrodynamic model was first presented by Gardner [15]. Therefore, it is natural to prove the existence of a global weak solution of (1.1)-(1.3). In the following, we reduce the number of equations $(1.1)^{\prime}-(1.3)^{\prime}$ by using a Green's function. 
The solution of the Poisson equation (1.3)' and the boundary data (1.9) is given uniquely by

$$
\phi=\int_{0}^{1} G(x, \xi)(\rho(\xi, t)-D(\xi)) d \xi+\phi_{1}+x\left(\phi_{2}-\phi_{1}\right),
$$

where $G(x, \xi)$ is Green's function for this problem and is defined by

$$
G(x, \xi)= \begin{cases}x(\xi-1), & x<\xi \\ \xi(x-1), & x>\xi\end{cases}
$$

From (1.10), we have

$$
\phi_{x}=\int_{0}^{1} G_{x}(x, \xi)(\rho-D(\xi)) d \xi+\phi_{2}-\phi_{1} .
$$

Then the system $(1.1)^{\prime}-(1.3)^{\prime}$ reduces to the following system:

$$
\begin{gathered}
\rho_{t}+m_{x}=0 \\
m_{t}+\left(\frac{m^{2}}{\rho}+p(\rho)\right)_{x}=\rho\left(\int_{0}^{1} G_{x}(\rho-D) d \xi+\phi_{2}-\phi_{1}\right)-\frac{m}{\tau} .
\end{gathered}
$$

The system (1.13)-(1.14) is a set of Euler equations of nonconservative form with two sources; i.e., an electric force and a collision term. Notice that the first source term, which is caused by the field $\phi_{x}$, is a nonlocal term involving some global properties of the solution of (1.13)-(1.14). In the following arguments, we choose the applied bias $\phi_{1}=\phi_{2}$, but this does not influence the outcome of the mathematical analysis.

As usual, to solve a nonlinear problem such as (1.13)-(1.14), the strategy is as follows: after introducing a suitable sequence of approximate solutions, we need enough a priori estimates to ensure the convergence of a subsequence to a solution. For problem (1.13)-(1.14), it is natural to consider the approximate solutions satisfying a Helly framework; i.e., approximate solutions that have uniform control on both spatial $L^{\infty}$ and total variation norms. However, it is difficult to show that the approximate solutions derived by the viscosity method satisfy this framework, since the principle of invariant regions or the weak maximum principle is hard to use due to the nonlocal term of (1.14). Also, this framework is not satisfied for the approximate solutions derived by the Lax-Friedrichs or Godunov scheme based on the analysis by [21]. The reason is that (1.13)-(1.14) loses its strict hyperbolicity in the vacuum state $\rho=0$. This is an essential feature of (1.13)-(1.14).

To overcome the above difficulties, Di Perna [11,12] and Chen [4] made a detailed analysis and established some framework theorems for hyperbolic conservation laws by using the theory of compensated compactness $[23,28]$. Di Perna obtained such a compactness framework for the viscosity method applied to the homogeneous system corresponding to (1.13)-(1.14) for $\gamma=1+\frac{2}{2 n+1}$ (integers $n \geqq 2$ ) [11]. And Chen generalized this compactness framework (such as Theorem 2.6 below) in the case $1<\gamma \leqq \frac{5}{3}$ [4]. The crucial idea of the proof of Theorem 2.6 is to show that a family of Young measures corresponding to uniformly bound approximate solutions reduces to a family of Dirac measures. 
One achieves this goal by showing that a family of entropy dissipation measures lies in a compact subset of the Sobolev space $\mathrm{H}_{\text {loc }}^{-1}$ for every weak entropy pair. For more details, see [4], [11], and [12]. This compactness framework has many advantages:

(1) The difficulties caused by $\rho=0$ can be overcome by virtue of the detailed analysis of the weak entropy-entropy flux pair.

(2) The complicated analysis of wave interactions can be avoided.

For example, the piston problem discussed by Nishida and Smoller [24] is very complicated, mainly as a result of the reflection of shock waves at the boundaries, where the strength of the reflected shock is greater than that of the incoming shock.

Definition 1.1. For every $T>0$, we define a weak solution of (1.13)-(1.14) to be a pair of bounded measurable functions $v(x, t)=(\rho(x, t), m(x, t))$ satisfying the following pair of integral identities:

$$
\begin{gathered}
\int_{0}^{T} \int_{0}^{1}\left(\rho \psi_{t}+m \psi_{x}\right) d x d t+\int_{t=0} \rho_{0} \psi d x=0, \\
\int_{0}^{T} \int_{0}^{1}\left(m \psi_{t}+\left(\frac{m^{2}}{\rho}+p(\rho)\right) \psi_{x}\right) d x d t \\
+\int_{0}^{T} \int_{0}^{1}\left(\rho \int_{0}^{1} G_{x}(\rho-D) d \xi-\frac{m}{\tau}\right) \psi d x d t+\int_{t=0} m_{0} \psi d x=0,
\end{gathered}
$$

for all $\psi \in C_{0}^{\infty}\left(I_{T}\right)$ satisfying $\psi(x, T)=0$ for $0 \leqq x \leqq 1$ and $\psi(0, t)=\psi(1, t)=0$ for $t \geqq 0$, where $I_{T}=(0,1) \times(0, T)$.

The following theorem is the main result of this paper.

Theorem 1.1. Suppose that the initial data $\left(\rho_{0}, m_{0}\right)$ and the given function $D(x)$ and $\tau$ satisfy the conditions

$$
\begin{gathered}
0 \leqq \rho_{0}(x) \leqq M_{1}, \quad \rho_{0}(x) \not \equiv 0, \quad\left|m_{0}(x)\right| \leqq M_{2} \rho_{0}(x), \\
|D(x)| \leqq M_{3}, \quad \text { and } 0<\tau_{0} \leqq \tau .
\end{gathered}
$$

for some positive constants $M_{i}(i=1,2,3)$ and $\tau_{0}$; and that $\tau$ satisfies the uniform Lipschitz condition. Then, for $1<\gamma \leqq \frac{5}{3}$, the initial-boundary value problem (1.13)(1.14) has a global weak solution $(\rho(x, t), m(x, t))$ satisfying the following estimates and entropy condition:

$$
\begin{aligned}
& \quad 0 \leqq \rho \leqq C, \quad|m| \leqq C \rho \text { a.e. for a constant } C>0, \text { and } \\
& \int_{0}^{T} \int_{0}^{1}\left(\eta(\rho, m) \tilde{\psi}_{t}+q(\rho, m) \tilde{\psi}_{x}\right) d x d t \\
& \quad+\int_{0}^{T} \int_{0}^{1} \eta_{m}(\rho, m)\left(\rho \int_{0}^{1} G_{x}(\rho-D) d \xi-\frac{m}{\tau}\right) \tilde{\psi} d x d t \geqq 0,
\end{aligned}
$$

for all weak and convex entropy pairs $(\eta, q)$ for (1.13)-(1.14) and for all nonnegative smooth functions $\tilde{\psi}$ that have compact support in the region $I_{T}$.

Moreover, the potential $\phi$ is given by (1.10).

The proof of this result mainly follows Chen [4], Ding et al. [8,9], and Di Perna $[11,12]$. In Sect. 2 we introduce some basic results which are used in 
subsequent sections. A sequence of approximate solutions $v_{h}$ derived by the Godunov scheme [17] is defined in Sect. 3. The $L^{\infty}$ norm of approximate solutions is estimated in Sect. 4. Although the principle of invariant regions cannot be applied directly (since $v_{h}$ are not pure Riemann solutions), it is noted that the approximate solutions cannot escape the Riemann invariant region by too much at each time step. The uniform bound of $v_{h}$ is then obtained by using this fact and a global property of the Riemann solutions. A compact embedding technique is used in Sect. 5 to see that the sequence of entropy dissipation measures $\eta\left(v_{h}\right)_{t}+q\left(v_{h}\right)_{x}$ lies in a compact subset of $H_{\text {loc }}^{-1}$. Then we can obtain Theorem 5.6; i.e., the framework of the approximate solutions. In Sect. 6 we use the result of [4] to get a subsequence, still labeled $v_{h}$, such that $v_{h}(x, t) \rightarrow(\rho(x, t), m(x, t))$ a.e. We then show that $(\rho, m)$ is a physical weak solution; i.e., it satisfies (1.15)-(1.18).

Most of the important cases in the hydrodynamic model for semiconductor devices, such as $\frac{5}{3}<\gamma \leqq 3$ in (1.4), the full one-dimensional hydrodynamic model, and the higher dimensional model for semiconductor devices, are still open. We believe that new methods must be developed to attack these nonlinear problems.

\section{Preliminaries}

In this section we first introduce some basic facts about the homogeneous system of equations (1.13)-(1.14). For more details, see [8,9], [18, 19], and [24, 26].

The homogeneous system corresponding to system of equations (1.13)-(1.14) reads:

$$
\begin{gathered}
\rho_{t}+m_{x}=0, \\
m_{t}+\left(\frac{m^{2}}{\rho}+p(\rho)\right)_{x}=0 .
\end{gathered}
$$

For a smooth solution, (2.1)-(2.2) can be written

$$
v_{t}+\nabla f(v) v_{x}=0
$$

where $v=(\rho, m)^{T}, f(v)=\left(m, \frac{m^{2}}{\rho}+\frac{\rho^{\gamma}}{\gamma}\right)^{T}$, and

The eigenvalues of (2.3) are

$$
\nabla f=\left(\begin{array}{cc}
0 & 1 \\
-\frac{m^{2}}{\rho^{2}}+\rho^{\gamma-1} & \frac{2 m}{\rho}
\end{array}\right) .
$$

$$
\lambda_{1}=\frac{m}{\rho}-\rho^{\theta}, \quad \lambda_{2}=\frac{m}{\rho}+\rho^{\theta},
$$

and the Riemann invariants are

$$
w=\frac{m}{\rho}+\frac{\rho^{\theta}}{\theta}, \quad z=\frac{m}{\rho}-\frac{\rho^{\theta}}{\theta},
$$

where $\theta=\frac{\gamma-1}{2}$. 
For the Riemann problem

$$
\left\{\begin{array}{cc}
(2.1)-(2.2), & t>0, \quad x \in \mathbb{R}, \\
\left.(\rho, m)\right|_{t=0}= \begin{cases}\left(\rho_{l}, m_{l}\right), & x<0, \\
\left(\rho_{r}, m_{r}\right), & x>0,\end{cases}
\end{array}\right.
$$

where $\rho_{l}, \rho_{r}, m_{l}$, and $m_{r}$ are constants satisfying $0 \leqq \rho_{l}, \rho_{r},\left|\frac{m_{l}}{\rho_{l}}\right|,\left|\frac{m_{r}}{\rho_{r}}\right|<\infty$, there are two distinct types of rarefaction waves and shock waves, called elementary waves, which are labeled 1-rarefaction or 2-rarefaction waves and 1-shock or 2-shock waves, respectively.

Lemma 2.1. There exists a global weak solution of (2.6) which is piecewise smooth function satisfying

$$
\begin{aligned}
& w(x, t) \equiv w(\rho(x, t), m(x, t)) \leqq \max \left\{w\left(\rho_{l}, m_{l}\right), w\left(\rho_{r}, m_{r}\right)\right\}, \\
& z(x, t) \equiv z(\rho(x, t), m(x, t)) \geqq \min \left\{z\left(\rho_{l}, m_{l}\right), z\left(\rho_{r}, m_{r}\right)\right\}, \\
& w(x, t)-z(x, t) \geqq 0 .
\end{aligned}
$$

It follows that the region $\Lambda=\left\{(\rho, m): w \leqq w_{0}, z \geqq z_{0}, w-z \geqq 0\right\}$ is an invariant region for the Riemann problem (2.6). More precisely, if the Riemann data lies in $\Lambda$, then the solution of (2.6) lies in $A$, too.

Lemma 2.2. If $\{(\rho, m): a \leqq x \leqq b\} \subset \Lambda$, then

$$
\left(\frac{1}{b-a} \int_{a}^{b} \rho d x, \frac{1}{b-a} \int_{b}^{a} m d x\right) \in \Lambda \text {. }
$$

Lemma 2.3. For the mixed problem

$$
\left\{\begin{array}{cll}
(2.1)-(2.2), & t>0, & x>0, \\
\left.(\rho, m)\right|_{t=0}=\left(\rho_{0}, m_{0}\right), & & x>0, \\
\left.m\right|_{x=0}=m_{1}, & t \geqq 0, &
\end{array}\right.
$$

where $\left(\rho_{0}, m_{0}\right)$ and $m_{1}$ are constants, there exists a weak solution in the region $\{(x, t): x \geqq 0, t \geqq 0\}$ satisfying the following estimates

$$
\begin{aligned}
& w(x, t) \leqq \max \left\{w\left(\rho_{0}, m_{0}\right), \frac{2 m_{1}}{\rho_{1}}-z\left(\rho_{0}, m_{0}\right)\right\}, \\
& z(x, t) \geqq z\left(\rho_{0}, m_{0}\right), \text { and } \quad w(x, t)-z(x, t) \geqq 0 .
\end{aligned}
$$

The term $\frac{2 m_{1}}{\rho_{1}}-z_{0}$ is new to the mixed problem because of shock waves reflecting off or coming out at the boundary $x=0$. As in (2.8), we can solve the following mixed problem in the region $\{(x, t): x \leqq 1, t \geqq 0\}$ :

$$
\left\{\begin{array}{cll}
(2.1)-(2.2), & t>0, & x<1, \\
\left.(\rho, m)\right|_{t=0}=\left(\rho_{0}, m_{0}\right), & & x<1, \\
\left.m\right|_{x=1}=m_{2}, & t \geqq 0 . &
\end{array}\right.
$$


The weak solution of (2.9) satisfies the following estimates:

$$
\begin{aligned}
& z(x, t) \geqq \min \left\{z\left(\rho_{0}, m_{0}\right), \frac{2 m_{2}}{\rho_{2}}-w\left(\rho_{0}, m_{0}\right)\right\}, \\
& w(x, t) \leqq w\left(\rho_{0}, m_{0}\right), \text { and } w(x, t)-z(x, t) \geqq 0 .
\end{aligned}
$$

Lemma 2.4. Suppose that $(\rho(x, t), m(x, t))$ is a solution of (2.6) or (2.8). Then, the jump strength of $m(x, t)$ across an elementary wave can be dominated by that of $\rho(x, t)$ across the same elementary wave, i.e.,

across a shock wave: $\quad\left|m_{r}-m_{l}\right| \leqq C\left|\rho_{r}-\rho_{l}\right|$,

across a rarefaction wave: $\quad\left|m-m_{l}\right| \leqq C\left|\rho-\rho_{l}\right| \leqq C\left|\rho_{r}-\rho_{l}\right|$,

where $C$ depends only on the bounds of $\rho$ and $|m|$.

Lemma 2.5. For any $\varepsilon>0$, there exist constants $h>0$ and $k>0$ such that the solution of (2.6) in the region $\{(x, t):|x|<h, 0 \leqq t<k\}$ satisfies

$$
\int_{-h}^{h}|\rho(x, t)-\rho(x, 0)| d x \leqq C h \varepsilon, \quad 0 \leqq t \leqq k,
$$

where $C$ depends only on the bounds of $\rho$ and $|m|$, and the mesh lengths $h$ and $k$ satisfy $\max _{i=1,2} \sup \left|\lambda_{i}(\rho, m)\right|<\frac{h}{2 k}$.

Definition 2.1. A pair of mappings $\eta: \mathbb{R}^{2} \rightarrow \mathbb{R}$ and $q: \mathbb{R}^{2} \rightarrow \mathbb{R}$ is called an entropyentropy flux pair if it satisfies the following equation

$$
\nabla q=\nabla \eta \nabla f
$$

Let $\tilde{\eta}\left(\rho, \frac{m}{\rho}\right)=\eta(\rho, m)$. If $\tilde{\eta}(0, u)=0$, then $\eta$ is called a weak entropy.

Among all entropies, the most natural entropy is the mechanical energy

$$
\eta_{*}(\rho, m)=\frac{1}{2} \frac{m^{2}}{\rho}+\frac{1}{\gamma(\gamma-1)} \rho^{\gamma},
$$

which plays a very important role in estimates for entropy dissipation measures. It is easy to check that $\eta_{*}$ is a weak and strictly convex entropy.

The following compactness framework was established in $[4,8]$.

Theorem 2.6. Assume that approximate solutions $v_{h}(x, t)=\left(\rho_{h}(x, t), m_{h}(x, t)\right)$ of the Cauchy problem (2.1)-(2.2) satisfy the following framework:

(1) $0 \leqq \rho_{h} \leqq C$ and $\left|m_{h}\right| \leqq C \rho_{h}$ a.e. for a positive constant $C$.

(2) The sequence of entropy dissipation measures $\eta\left(v_{h}\right)_{t}+q\left(v_{h}\right)_{x}$ is compact in $H_{\text {loc }}^{-1}(\Omega)$ for every weak entropy pair $(\eta, q)$ and every open bounded set $\Omega \subset \mathbb{R}_{+}^{2}$.

Then, for $1<\gamma \leqq \frac{5}{3}$, there exists a convergent subsequence, still labeled $v_{h}$, such that

$$
\left(\rho_{h}(x, t), m_{h}(x, t)\right) \rightarrow(\rho(x, t), m(x, t)) \quad \text { a.e. }
$$

For the proof of Theorem 2.6, see [4]. 


\section{The Godunov Scheme}

We choose the space mesh length $h=\frac{1}{N}$, where $N$ is a positive integer. The time mesh length $k=k(h)$ will be chosen later so that the Courant-Friedrich-Levy condition

$$
\max _{i=1,2}\left(\sup \left|\lambda_{i}(v)\right|\right)<\frac{h}{2 k}
$$

holds for a given $T>0$. We partition the interval $[0,1]$ into cells, with the $j^{\text {th }}$ cell centered at $x_{j}=j h, j=1, \ldots, N-1$. We now use the Godunov scheme to construct a sequence of approximate solutions of (1.13)-(1.14). Namely, we solve the Riemann problems (2.6) in the region $R_{j}^{1} \equiv\left\{(x, t): x_{j-\frac{1}{2}} \leqq x<x_{j+\frac{1}{2}}\right.$, $0 \leqq t<k\}$ :

$$
\left\{\begin{array}{l}
\frac{\partial}{\partial t} \underline{v}_{h}+\frac{\partial}{\partial x} f\left(\underline{v}_{h}\right)=0 \\
\left.\underline{v}_{h}\right|_{t=0}= \begin{cases}\left(\rho_{j}^{0}, m_{j}^{0}\right), & x<x_{j}, \\
\left(\rho_{j+1}^{0}, m_{j+1}^{0}\right), & x>x_{j}, \quad j=1, \ldots, N-1,\end{cases}
\end{array}\right.
$$

where

$$
\rho_{j}^{0}=\frac{1}{h} \int_{x_{j-1}}^{x_{j}} \rho_{0}(x) d x, \quad m_{j}^{0}=\frac{1}{h} \int_{x_{j-1}}^{x_{j}} m_{0}(x) d x, \quad \text { for } j=1, \ldots, N .
$$

We also solve the mixed problems (2.8) and (2.9) for $\left(\rho_{1}^{0}, m_{1}^{0}\right), m_{1}=0$, and $\left(\rho_{N}^{0}, m_{N}^{0}\right)$, $m_{2}=0$, in regions $R_{0}^{1} \equiv\left\{(x, t): 0 \leqq x<x_{\frac{1}{2}}, 0 \leqq t<k\right\}$ and $R_{N}^{1} \equiv\left\{(x, t): x_{N-\frac{1}{2}} \leqq x\right.$ $<1,0 \leqq t<k\}$, respectively. Then we set

$$
v_{h}(x, t)=\underline{v}_{h}(x, t)+V\left(\underline{v}_{h}(x, t)\right) t, \quad 0 \leqq x \leqq 1, \quad 0 \leqq t<k,
$$

where $V(v)=\left(V_{1}(v), V_{2}(v)\right) \equiv\left(0, \rho \int_{0}^{1} G_{x}(\rho-D) d \xi-\frac{m}{\tau}\right)$,

$$
v_{j}^{1}=\frac{1}{h} \int_{x_{j-1}}^{x_{j}} v_{h}\left(x, t_{1}-0\right) d x, \quad j=1, \ldots, N .
$$

Suppose that we have defined approximate solutions $v_{h}(x, t)$ for $0 \leqq t<t_{i}$. We then define

$$
v_{h}(x, t)=\underline{v}_{h}(x, t)+V\left(\underline{v}_{h}(x, t)\right)\left(t-t_{i}\right), \quad t_{i} \leqq t<t_{i+1},
$$

where $\underline{v}_{h}(x, t)$ are piecewise smooth functions defined as solutions of the Riemann problems in the region $R_{j}^{i+1}=\left\{(x, t): x_{j-\frac{1}{2}} \leqq x<x_{j+\frac{1}{2}}, t_{i} \leqq t<t_{i+1}\right\}$

$$
\left\{\begin{array}{l}
(2.1)-(2.2), \\
\left.\underline{v}_{h}\right|_{t=t_{t}}= \begin{cases}v_{j}^{i}, & x<x_{j}, \\
v_{j+1}^{i}, & x>x_{j}, \quad j=1, \ldots, N-1,\end{cases}
\end{array}\right.
$$


and as solutions of mixed problems in the two regions $R_{0}^{i}$ and $R_{N}^{i}$ :

(a)

$$
\begin{aligned}
R_{0}^{i}= & \left\{(x, t): 0 \leqq x<x_{\frac{1}{2}}, t_{i} \leqq t<t_{i+1}\right\}, \\
& \left\{\begin{array}{l}
(2.1)-(2.2), \quad x>0, \quad t>t_{i}, \\
\left.\underline{v}_{h}\right|_{t=t_{i}}=v_{1}^{i}, \quad x>0, \\
\left.\underline{m}_{h}\right|_{x=0}=0 .
\end{array}\right.
\end{aligned}
$$

$$
\begin{aligned}
R_{N}^{i}= & \left\{(x, t): x_{N-\frac{1}{2}} \leqq x<1, t_{i} \leqq t<t_{i+1}\right\}, \\
& \left\{\begin{array}{c}
(2.1)-(2.2), \quad x<1, \quad t>t_{n}, \\
\left.\underline{v}_{h}\right|_{t=t_{i}}=v_{N}^{i}, \quad x<1, \\
\left.\underline{m}_{h}\right|_{x=1}=0 .
\end{array}\right.
\end{aligned}
$$

Still, we can define the Godunov scheme

$$
v_{j}^{i+1}=\frac{1}{h} \int_{x_{j-1}}^{x_{j}} v_{h}\left(x, t_{i+1}-0\right) d x, \quad 1 \leqq j \leqq N .
$$

The approximate solutions $v_{h}=\left(\rho_{h}, m_{h}\right) \equiv\left(\underline{\rho}_{h}, m_{h}\right)$ are well-defined, since $\rho_{h} \geqq 0$. We summarize the above process as follows:

$$
v^{i+1}=A_{h} \circ R \circ E_{k}\left(\cdot, v^{i}\right),
$$

where $A_{h}$ is the cell-averaging operator (3.6), $E_{k}\left(x, v^{i}\right)$ is the exact evolution operator (3.5), and $R$ is the reconstruction step (3.4).

For $t_{i} \leqq t<t_{i+1}$, we set

$$
\begin{aligned}
& w_{h}(x, t)=\underline{w}_{h}(x, t)+\left\{\int_{0}^{1} G_{x}\left[\left(\frac{\theta\left(\underline{w}_{h}-\underline{z}_{h}\right)}{2}\right)^{\frac{1}{\theta}}-D\right] d \xi-\frac{\underline{w}_{h}+\underline{z}_{h}}{\tau}\right\}\left(t-t_{i}\right), \\
& z_{h}(x, t)=\underline{z}_{h}(x, t)+\left\{\int_{0}^{1} G_{x}\left[\left(\frac{\theta\left(\underline{w}_{h}-\underline{z}_{h}\right)}{2}\right)^{\frac{1}{\theta}}-D\right] d \xi-\frac{\underline{w}_{h}+\underline{z}_{h}}{\tau}\right\}\left(t-t_{i}\right),
\end{aligned}
$$

where $\underline{w}_{h}$ and $\underline{z}_{h}$ are Riemann invariants corresponding to the Riemann solutions $\underline{v}_{h}$.

\section{Uniform Bound for Approximate Solutions}

To obtain uniform bound for the approximate solutions, we estimate $w_{h}(x, t)$ and $z_{h}(x, t)$ defined by (3.8) and (3.9). Notice that (3.8)-(3.9) involve nonlocal terms such as $\int_{0}^{1} G_{x} \rho_{h} d \xi$. If we integrate (1.13) and use the initial and boundary data, we obtain the following conservation of particles:

$$
\int_{0}^{1} \rho(x, t) d x=\int_{0}^{1} \rho_{0}(x) d x, \quad \text { for } t \geqq 0 .
$$

As in (4.1), the approximate solutions $\rho_{h}(x, t)=\underline{\rho}_{h}(x, t)$ also satisfy this conservation principle. 
Lemma 4.1. Let $v_{h}=\left(\underline{\rho}_{h}, m_{h}\right)$ be the approximate solutions as defined above. Then,

$$
\int_{0}^{1} \underline{\rho}_{h}\left(x, t_{i+1}\right) d x=\int_{0}^{1} \rho_{0}(x) d x, \quad 0 \leqq i \leqq n-1,
$$

where the positive integer $n$ is defined later.

Proof. In the region $R_{i} \equiv\left\{(x, t): 0 \leqq x \leqq 1, t_{i} \leqq t<t_{i+1}\right\}$, the Riemann solutions $\underline{v}_{h}=\left(\varrho_{h}, \underline{m}_{h}\right)$ consist of piecewise smooth functions. Using the Green's formula, we have

$$
\int_{0}^{1} \underline{\rho}_{h}\left(x, t_{i+1}-0\right) d x+\int_{t_{i}}^{t_{i+1}} \sum\left\{\sigma\left[\underline{\rho}_{h}\right]-\left[\underline{m}_{h}\right]\right\} d t=\int_{0}^{1} \underline{\rho}_{h}\left(x, t_{i}+0\right) d x
$$

where the sumation $\sum$ is taken over all the shock waves in $\left(\rho_{h}, \underline{m}_{h}\right)$ at a fixed $t$ between $t_{i}$ and $t_{i+1} ; \sigma$ is the propagating speed of the shock wave; [ $\left.\rho_{h}\right]$ and $\left[\underline{m}_{h}\right]$ denote the jump of $\rho_{h}$ and $\underline{m}_{h}$ across the shock wave from left to right, respectively. That is, if $S=(x(t), t)$ denotes a shock in $\left(\underline{\rho}_{h}, \underline{m}_{h}\right)$, then

$$
\begin{aligned}
{\left[\underline{\rho}_{h}\right] } & =\underline{\rho}_{h}(x(t)+0, t)-\underline{\rho}_{h}(x(t)-0, t), \\
{\left[\underline{m}_{h}\right] } & =\underline{m}_{h}(x(t)+0, t)-\underline{m}_{h}(x(t)-0, t) .
\end{aligned}
$$

By the Rankine-Hugoniot condition, we have

$$
\int_{t_{i}}^{t_{i+1}} \sum\left\{\sigma\left[\underline{\rho}_{h}\right]-\left[\underline{m}_{h}\right]\right\} d t=0 .
$$

It follows from (4.3) and the definition of $\underline{\rho}_{h}$ that

$$
\int_{0}^{1} \underline{\rho}_{h}\left(x, t_{i+1}\right) d x=\int_{0}^{1} \underline{\rho}_{h}\left(x, t_{i}\right)
$$

and (4.2) holds.

Theorem 4.2. Suppose that the initial data $\left(\rho_{0}(x), m_{0}(x)\right)$ and the given function $D(x)$ satisfy the following conditions:

$$
0 \leqq \rho_{0}(x) \leqq M_{1}, \quad \rho_{0}(x) \not \equiv 0, \quad\left|m_{0}(x)\right| \leqq M_{2} \rho_{0}(x), \quad \text { and }|D(x)| \leqq M_{3} .
$$

Then, the approximate solutions $\left(\rho_{h}, m_{h}\right)$ derived by the Godunov scheme are uniformly bounded in the region $\bar{I}_{T} \equiv\{(x, t): 0 \leqq x \leqq 1,0 \leqq t \leqq T\}$; that is, there is a constant $C(T)>0$ such that

$$
0 \leqq \rho_{h}(x, t) \leqq C, \quad\left|m_{h}(x, t)\right| \leqq C \rho_{h}(x, t) .
$$

Proof. Assume that $0<k<2 \tau_{0}$. For $t_{i} \leqq t<t_{i+1}$ ( $i \geqq 0$ integers), the Riemann invariant properties and Lemma 4.1 imply that

$$
\begin{aligned}
w_{h}(x, t) & =\underline{w}_{h}(x, t)\left(1-\frac{t-t_{i}}{2 \tau}\right)+\left[\int_{0}^{1} G_{x}\left(\underline{\rho}_{h}-D\right) d \xi-\frac{\underline{z}_{h}(x, t)}{2 \tau}\right]\left(t-t_{i}\right) \\
& \leqq \sup _{x} \underline{w}_{h}\left(x, t_{i}+0\right)\left(1-\frac{t-t_{i}}{2 \tau}\right)-\inf _{x} \underline{z}_{h}\left(x, t_{i}+0\right) \frac{t-t_{i}}{2 \tau} \tilde{M} k
\end{aligned}
$$




$$
\begin{aligned}
z_{h}(x, t) & =\underline{z}_{h}(x, t)\left(1-\frac{t-t_{i}}{2 \tau}\right)+\left[\int_{0}^{1} G_{x}\left(\underline{\rho}_{h}-D\right) d \xi-\frac{\underline{w}_{h}(x, t)}{2 \tau}\right]\left(t-t_{i}\right) \\
& \geqq \inf _{x} \underline{z}_{h}\left(x, t_{i}+0\right)\left(1-\frac{t-t_{i}}{2 \tau}\right)-\sup _{x} \underline{w}_{h}\left(x, t_{i}+0\right) \frac{t-t_{i}}{2 \tau}-\tilde{M} k .
\end{aligned}
$$

Let $\alpha_{i}=\max \left\{\sup _{x} \underline{w}_{h}\left(x, t_{i}+0\right),-\inf _{x} \underline{z}_{h}\left(x, t_{i}+0\right)\right\}$. Then

$$
\max \left\{\sup _{x} \underline{w}_{h}\left(x, t_{i+1}-0\right),-\inf _{x} z_{h}\left(x, t_{i+1}-0\right)\right\} \leqq \alpha_{i}+\tilde{M} k .
$$

It follows that

$$
\begin{gathered}
\alpha_{i+1} \leqq \alpha_{i}+\tilde{M} k, \quad \text { and } \\
\alpha_{i} \leqq \alpha_{0}+\tilde{M} T, \quad 0 \leqq i \leqq n
\end{gathered}
$$

where $\alpha_{0}=\max \left\{\sup _{x} w_{0}(x),-\inf _{x} z_{0}(x)\right\}$. Then,

$$
\begin{aligned}
& w_{h}(x, t) \leqq \alpha_{0}+\tilde{M} T, \quad z_{h}(x, t) \geqq-\alpha_{0}-\tilde{M} T, \quad \text { and } \\
& w_{h}(x, t)-z_{h}(x, t) \geqq 0 .
\end{aligned}
$$

Then there is a constant $C(T)>0$ independent of $h$ and $k$ such that

$$
0 \leqq \rho_{h}(x t) \leqq C, \quad\left|m_{h}(x, t)\right| \leqq C \rho_{h}(x, t) .
$$

Now, we can choose the time mesh length $k=k(h)$. Let

$$
\lambda=\max _{i=1,2}\left\{\sup _{\substack{0 \leq \rho \leq C \\|m| \leqq C}}\left|\lambda_{i}(\rho, m)\right|\right\}
$$

then we take

$$
k=\frac{T}{n}, \quad \text { where } \quad n=\max \left\{\left[\frac{4 \lambda T}{h}\right]+1,\left[\frac{T}{2 \tau_{0}}\right]+1\right\} .
$$

For this $k$, the CFL condition and $0<k<2 \tau_{0}$ hold.

\section{Compactness of Entropy Dissipation Measures}

In this section, we estimate the $H_{\text {loc }}^{-1}$-compactness of entropy dissipation measures $\eta\left(v_{h}\right)_{t}+q\left(v_{h}\right)_{x}$ associated with weak entropy pair $(\eta, q)$ and approximate solutions of the Godunov scheme. To achieve this goal, we follow the technique used in [8].

First, we estimate the mechanical entropy pair which dominates all other weak entropy pairs. Second, by duality and the Sobolev interpolation inequality, we show the $W^{-1, p}$ compactness and estimate the $W^{-1, r}$ bound of entropy dissipation measures for some $1<p \leqq 2<r<\infty$, instead of $H_{\text {loc }}^{-1}$ compactness. For simplicity, we drop the subindex $h$ of approximate solutions $v_{h}$ and the Riemann solutions $\underline{v}_{h}$ in the following arguments.

Lemma 5.1. Let $v$ be the approximate solution defined in Sect. 3. Then, there is a positive constant $C$ independent of $h$ such that

$$
\sum_{i, j} \int_{x_{j}-1}^{x_{j}}\left|v\left(x, t_{i}-0\right)-v_{j}^{i}\right|^{2} d x \leqq C .
$$


Proof. Consider the mechanical entropy pair

$$
\eta_{*}=\frac{1}{2} \frac{m^{2}}{\rho}+\frac{\rho^{\gamma}}{\gamma(\gamma-1)}, \quad q_{*}=\frac{1}{2} \frac{m^{3}}{\rho^{2}}+\frac{1}{\gamma-1} \rho^{\gamma-1} m .
$$

For $t_{i} \leqq t \leqq t_{i+1}$, Green's formula implies that

$$
\sum_{j=1}^{N} \int_{x_{j-1}}^{x_{j}}\left(\eta_{*}\left(\underline{v} \frac{i+1}{-}\right)-\eta_{*}\left(v_{j}^{i}\right)\right) d x+\int_{t_{i}}^{t_{t}} \sum\left\{\sigma\left[\eta_{*}\right]-\left[q_{*}\right]\right\} d t=0,
$$

where $\underline{v} \frac{i+1}{=} \underline{v}\left(x, t_{i+1}-0\right)$, the summation $\sum$ and notation $\sigma$ are similar to those of Lemma 4.1, and

$$
\begin{aligned}
& {\left[\eta_{*}\right]=\eta_{*}(\underline{v}(x(t)+0, t))-\eta_{*}(\underline{v}(x(t)-0, t)),} \\
& {\left[q_{*}\right]=q_{*}(\underline{v}(x(t)+0, t))-q_{*}(\underline{v}(x(t)-0, t)), \quad t_{i} \leqq t<t_{i+1} .}
\end{aligned}
$$

Summing over all $i$ in (5.2) gives:

$$
\begin{gathered}
\sum_{\substack{1 \leqq i \leqq n-1 \\
1}} \int\left(\eta_{*}\left(\underline{v}^{i}\right)-\eta_{*}\left(v_{j}^{i}\right)\right) d x+\int_{0}^{T} \sum\left\{\sigma\left[\eta_{*}\right]-\left[q_{*}\right]\right\} d t \\
=\int_{0}^{1}\left(\eta_{*}\left(\underline{v}_{0}(x)\right)-\eta_{*}(\underline{v}(x, T-0))\right) d x
\end{gathered}
$$

where $\int=\int_{x_{j-1}}^{x_{j}}$ and $\underline{v}-\left(x, t_{i}-0\right)$. From the uniform bound of $\underline{v}$, the right side of (5.3) is dominated by a constant $C>0$ independent of $h$; i.e.,

$$
\sum_{i, j} \int\left(\eta_{*}\left(\underline{v}^{i}\right)-\eta_{*}\left(v_{j}^{i}\right)\right) d x+\int_{0}^{T} \sum\left\{\sigma\left[\eta_{*}\right]-\left[q_{*}\right]\right\} d t \leqq C .
$$

On the cell $\left(x_{j-1}, x_{j}\right)$, we decompose the first term of (5.4) into two parts:

$$
\begin{aligned}
& \int_{x_{j-1}}^{x_{j}}\left(\eta_{*}\left(\underline{v}^{-}\right)-\eta_{*}\left(v_{j}^{i}\right)\right) d x \\
& \quad=\int_{x_{j-1}}^{x_{j}}\left(\eta_{*}\left(v^{-}\right)-\eta_{*}\left(v_{j}^{i}\right)\right) d x+\int_{x_{j-1}}^{x_{j}}\left(\eta_{*}\left(\underline{v}^{-}\right)-\eta_{*}\left(v^{-}\right)\right) d x \\
& \quad \equiv A_{j}^{i}+R_{j}^{i},
\end{aligned}
$$

where $v_{-}^{i}=v\left(x, t_{i}-0\right)$. Notice that $A_{j}^{i}$ and $R_{j}^{i}$ are caused by the cell-averaging operator and reconstruction step, respectively.

For $A_{j}^{i}$, we take the Taylor expansion for $\eta_{*}\left(v^{i}\right)$ to get

$$
\eta_{*}\left(v_{-}^{i}\right)=\eta_{*}\left(v_{j}^{i}\right)+\nabla \eta_{*}\left(v_{j}^{i}\right)\left(v_{-}^{i}-v_{j}^{i}\right)+\frac{1}{2}\left(v_{-}^{i}-v_{j}^{i}\right)^{T} \nabla^{2} \eta_{*}\left(\xi_{j}^{i}\right)\left(v_{-}^{i}-v_{-j}^{i}\right),
$$

where $\xi_{j}^{i}$ is a mean value. Integrating (5.5) on the cell and using the fact that $v_{j}^{i}$ is the average value of $v^{i}$ on this cell, we have

$$
A_{j}^{i}=\frac{1}{2} \int_{x_{j-1}}^{x_{j}}\left(v_{-}^{i}-v_{j}^{i}\right)^{T} \nabla^{2} \eta_{*}\left(\xi_{j}^{i}\right)\left(v^{i}-v_{j}^{i}\right) d x .
$$


For $R_{j}^{i}$, we have

$$
\begin{aligned}
R_{j}^{i} & =\int_{x_{\jmath}-1}^{x_{j}}\left[\int_{0}^{1} \nabla \eta_{*}\left(v^{\underline{i}}+\theta\left(\underline{v}^{\underline{i}}-v^{\underline{i}}\right)\right) d \theta\left(\underline{v}^{\underline{i}}-v^{-}\right)\right] d x \\
& =-k \int_{x_{j-1}}^{x_{j}}\left[\int_{0}^{1} \nabla \eta_{*}\left(v^{\underline{i}}+\theta\left(\underline{v}^{\underline{i}}-v^{\underline{i}}\right)\right) d \theta V\left(\underline{v}^{-}\right)\right] d x .
\end{aligned}
$$

From the uniform bound of approximate solutions, we get

$$
\left|R_{j}^{i}\right| \leqq C h k \text {, where } C \text { is independent of } h \text { and } k \text {. }
$$

Summing over all cells, by (5.4), (5.6), and (5.7), we have

$$
\sum_{i, j} \int\left(v^{i}-v_{j}^{i}\right)^{T} \nabla^{2} \eta_{*}\left(\xi_{j}^{i}\right)\left(v^{i}-v_{j}^{i}\right) d x+2 \int_{0}^{T} \sum\left\{\sigma\left[\eta_{*}\right]-\left[q_{*}\right]\right\} d t \leqq C .
$$

Since $\left(\eta_{*}, q_{*}\right)$ is a convex entropy pair, the point entropy $\sigma\left[\eta_{*}\right]-\left[q_{*}\right] \geqq 0$ holds across the shock waves [8]. It follows from (5.8) that

$$
\begin{gathered}
\int_{0}^{T} \sum\left\{\sigma\left[\eta_{*}\right]-\left[q_{*}\right]\right\} d t \leqq C, \\
\sum_{i, j} \int\left(v^{i}-v_{-j}^{i}\right)^{T} \nabla^{2} \eta_{*}\left(\xi_{j}^{i}\right)\left(v^{i}-v_{j}^{i}\right) d x \leqq C .
\end{gathered}
$$

In particular, $\eta_{*}$ is strictly convex; i.e., there is a constant $\alpha>0$ such that $v^{T} \nabla^{2} \eta_{*} v \geqq \alpha|v|^{2}$. It follows from (5.10) that (5.1) is true.

The proof of the following three lemmas can be found in [8] and [11].

Lemma 5.2 [11]. Assume that $0 \leqq \rho \leqq C,|m| \leqq C \rho$. Then, there is a constant $\bar{C}>0$ such that

$$
\begin{gathered}
|\nabla \eta| \leqq \bar{C}, \quad|\nabla q| \leqq \bar{C} \\
\left|v^{T} \nabla^{2} \eta v\right| \leqq \bar{C} v^{T} \nabla^{2} \eta_{*} v
\end{gathered}
$$

for every weak entropy pair $(\eta, q)$.

Lemma 5.3 [8]. For every weak entropy pair $(\eta, q)$, there is a constant $C>0$ such that

$$
|\sigma[\eta]-[q]| \leqq C\left\{\sigma\left[\eta_{*}\right]-\left[q_{*}\right]\right\} .
$$

Lemma 5.4 [8]. Let $\Omega \subset \mathbb{R}^{m}$ be a bounded open set. Then, (compact set of $\left.W^{-1, p}(\Omega)\right)$ $\cap\left(\right.$ bounded set of $W^{-1, r}(\Omega) \subset\left(\right.$ compact set of $\left.H_{\mathrm{loc}}^{-1}(\Omega)\right)$ for some constants $p$ and $r$ satisfying $1<p \leqq 2<r<\infty$.

Now, we can prove that the sequence of entropy dissipation measures $\eta(v)_{t}+q(v)_{x}$ is compact in $H_{\text {loc }}^{-1}$.

Theorem 5.5. Assume that the conditions of Theorem 4.2 are satisfied. Then,

$$
\text { the set }\left\{\eta(v)_{t}+q(v)_{x}\right\} \text { of measures is compact in } H_{\mathrm{loc}}^{-1}(\Omega) \text {, }
$$

for every weak entropy pair $(\eta, q)$ and every open subset $\Omega \subset \bar{I}_{T}$. 
Proof. For any $\psi \in C_{0}^{\infty}(\Omega)$, we consider

$$
\int_{0}^{T} \int_{0}^{1}\left(\eta(v) \psi_{t}+q(v) \psi_{x}\right) d x d t=A(\psi)+R(\psi)+B(\psi)+\Sigma(\psi)+S(\psi),
$$

where

$$
\begin{aligned}
A(\psi) & =\sum_{i, j} \int\left(\eta\left(v^{\underline{i}}\right)-\eta\left(v_{j}^{i}\right)\right) \psi\left(x, t_{i}\right) d x, \\
R(\psi) & =\sum_{i, j} \int\left(\eta\left(\underline{v}^{i}\right)-\eta\left(v^{i}\right)\right) \psi\left(x, t_{i}\right) d x, \\
B(\psi) & =\int_{0}^{1}[\eta(\underline{v}(x, T)) \psi(x, T)-\eta(\underline{v}(x, 0)) \psi(x, 0)] d x, \\
\Sigma(\psi) & =\int_{0}^{T} \sum\{\sigma[\eta]-[q]\} \psi(x(t), t) d t, \\
S(\psi)= & \int_{0}^{T} \int_{0}^{1}\left[(\eta(v)-\eta(\underline{v})) \psi_{t}+(q(v)-q(\underline{v})) \psi_{x}\right] d x d t .
\end{aligned}
$$

Observe that the entropy dissipation measures $\eta(v)_{t}+q(v)_{x}$ are mainly supported on the shock of the Riemann solutions $\underline{v}$ in the region $I \times\left(t_{i}, t_{i+1}\right)$, $0 \leqq i \leqq n-1$, and on the interfacial lines $I \times\left\{t_{i}\right\}, 1 \leqq i \leqq n-1$. The term $\Sigma(\psi)$ is caused by the shocks in $\underline{v}, S(\psi)$ by the reconstruction step; $A(\psi)$ and $R(\psi)$ are caused by the cell-averaging operator and reconstruction step on all discrete time steps $t_{i}$, respectively. In the following, we estimate them one by one.

(a) We decompose $A(\psi)$ into two parts:

$$
\begin{aligned}
A(\psi)= & \sum_{i, j} \psi_{j}^{i} \int\left(\eta\left(v^{i}\right)-\eta\left(v_{j}^{i}\right)\right) d x \\
& +\sum_{i, j} \int\left(\eta\left(v^{i}\right)-\eta\left(v_{j}^{i}\right)\right)\left(\psi^{i}-\psi_{j}^{i}\right) d x \\
\equiv & A_{1}(\psi)+A_{2}(\psi),
\end{aligned}
$$

where $\psi_{j}^{i}=\psi\left(x_{j}, t_{i}\right)$ and $\psi^{i}=\psi\left(x, t_{i}\right)$.

For $A_{1}(\psi)$, using (5.5)-(5.6), (5.10), and (5.12), we have

$$
\begin{aligned}
\left|A_{1}(\psi)\right| & =\frac{1}{2}\left|\sum_{i, j} \psi_{j}^{i} \int\left(v^{i}-v_{j}^{i}\right)^{T} \nabla^{2} \eta\left(\xi_{j}^{i}\right)\left(v^{\underline{i}}-v_{j}^{i}\right) d x\right| \\
& \leqq C\|\psi\|_{\infty} \sum_{i, j} \int\left(v^{i}-v_{j}^{i}\right)^{T} \nabla^{2} \eta_{*}\left(\xi_{j}^{i}\right)\left(v^{\underline{i}}-v_{j}^{i}\right) d x \\
& \leqq C\|\psi\|_{\infty} .
\end{aligned}
$$

For $A_{2}(\psi)$, using Hölder's inequality, (5.1), and (5.11), we have

$$
\begin{aligned}
\left|A_{2}(\psi)\right| & \leqq\left(\sum_{i, j} \int\left(\psi^{i}-\psi_{j}^{i}\right)^{2} d x\right)^{\frac{1}{2}}\left(\sum_{i, j} \int\left(\eta\left(v^{\underline{i}}\right)-\eta\left(v_{j}^{i}\right)\right)^{2} d x\right)^{\frac{1}{2}} \\
& \leqq\left(\frac{h}{2}\right)^{\alpha-\frac{1}{2}}\|\psi\|_{c_{0}^{\alpha}}\left(\sum_{i, j} \int\left|x-x_{j}\right| d x\right)^{\frac{1}{2}}\left(\sum_{i, j} \int\left|\nabla \eta\left(v^{\underline{i}}-v_{j}^{i}\right)\right|^{2} d x\right)^{\frac{1}{2}}
\end{aligned}
$$




$$
\begin{aligned}
& \leqq C h^{\alpha-\frac{1}{2}}\|\nabla \eta\|_{\infty}\|\psi\|_{C_{0}^{\alpha}}\left(\sum_{i, j} \int\left(v^{i}-v_{j}^{i}\right)^{2} d x\right)^{\frac{1}{2}} \\
& \leqq C h^{\alpha-\frac{1}{2}}\|\psi\|_{C_{0}^{\alpha}}
\end{aligned}
$$

where $\frac{1}{2}<\alpha<1$.

(b) We estimate the term $R(\psi)$, which is a result of the reconstruction step, by using (5.11) and the uniform bound of $v$, so that

$$
\begin{aligned}
|R(\psi)| & \leqq \sum_{i, j} \int\left|\nabla \eta\left(\xi_{j}^{i}\right)\left(\underline{v}^{\underline{i}}-v^{i}\right) \psi^{i}\right| d x \\
& \leqq k\|\nabla \eta\|_{\infty}\|\psi\|_{\infty} \sum_{i, j} \int \mid V\left(\left(\underline{v}^{-}\right) \mid d x\right. \\
& \leqq C\|V(\underline{v})\|_{\infty}\|\psi\|_{\infty} \sum_{i, j} h k \leqq C\|\psi\|_{\infty} .
\end{aligned}
$$

(c) It is easy to see that

$$
|B(\psi)| \leqq\|\psi\|_{\infty} \int_{0}^{1}\left(\mid \eta\left(\underline{v}(x, T)|+| \eta(\underline{v}(x, 0) \mid) d x \leqq C\|\psi\|_{\infty} .\right.\right.
$$

(d) It follows from (5.9) and (5.13) that

$$
\begin{aligned}
|\Sigma(\psi)| & \leqq\|\psi\|_{\infty} \int_{0}^{T} \sum|\sigma[\eta]-[q]| d t \\
& \leqq C\|\psi\|_{\infty} \int_{0}^{T} \sum\left\{\sigma\left[\eta_{*}\right]-\left[q_{*}\right]\right\} d t \leqq C\|\psi\|_{\infty} .
\end{aligned}
$$

(e) It follows from (5.11) that

$$
\begin{aligned}
|S(\psi)| & \leqq \int_{0}^{T} \int_{0}^{1}\left(\left|\nabla \eta\left(\xi_{1}\right)\right|\left|\psi_{t}\right|+\left|\nabla q\left(\xi_{2}\right)\right|\left|\psi_{x}\right|\right)|v-\underline{v}| d x d t \\
& \leqq k\left\|V_{2}(\underline{v})\right\|_{\infty}\left(\|\nabla \eta\|_{\infty}+\|\nabla q\|_{\infty}\right) \int_{0}^{T} \int_{0}^{1}\left(\left|\psi_{t}\right|+\left|\psi_{x}\right|\right) d x d t \\
& \leqq C h\|\psi\|_{H_{0}^{1}(\Omega)} .
\end{aligned}
$$

Since $C_{0}^{\infty}(\Omega)$ is dense in $H_{0}^{1}(\Omega)$, it follows that

$$
\|S\|_{H_{\mathrm{loc}}^{-1}(\Omega)} \leqq C h \rightarrow 0 \quad \text { as } h \rightarrow 0 \text {. }
$$

Thus,

$$
S \text { is compact in } H_{\text {loc }}^{-1}(\Omega) \text {. }
$$

Using the above estimates, we can apply Lemma 5.4 to get the compactness in $H_{\text {loc }}^{-1}(\Omega)$. First, by (5.22) and (5.24)-(5.26), we have

$$
\left\|A_{1}+R+B+\Sigma\right\|_{\left(C_{0}\right)^{*}} \leqq C
$$

By the embedding theorem, $\left(C_{0}(\Omega)\right)^{*} \hookrightarrow W^{-1, p_{0}}$ is compact for $1<p_{0}<2$. Thus,

$$
A_{1}+R+B+\sum \text { is compact in } W^{-1, p_{0}}(\Omega) \text {. }
$$


By the Sobolev theorem, $W_{0}^{1, p_{1}}(\Omega) \subset C_{0}^{\beta}(\Omega)$, for $0<\beta<1-\frac{2}{p_{1}}$, and the estimate (5.23), we have

$$
\left|A_{2}(\psi)\right| \leqq C h^{\alpha-\frac{1}{2}}\|\psi\|_{W_{0}^{1, p_{1}}(\Omega)} \quad \text { for } p_{1}>\frac{2}{1-\alpha}
$$

It follows from duality that

$$
\left\|A_{2}\right\|_{W^{-1, p_{2}}(\Omega)} \leqq C h^{\alpha-\frac{1}{2}} \rightarrow 0 \quad \text { as } h \rightarrow 0 \text { for } 1<p_{2}<\frac{2}{1+\alpha} .
$$

Then,

$$
A_{2} \text { is compact in } W^{-1, p_{2}}(\Omega) .
$$

Combining (5.29) and (5.30), we see that

$$
A+R+B+\Sigma=A_{1}+A_{2}+R+B+\Sigma \text { is compact in } W^{-1, p}(\Omega),
$$

where $1<p<\min \left(p_{0}, p_{2}\right)$.

Next, from the uniform bound of $v$, we have the following fact:

$$
\eta(v)_{t}+q(v)_{x}-S \text { is bounded in } W^{-1, \infty}(\Omega) .
$$

Since $\Omega$ is bounded, the above statement implies that

$$
\eta(v)_{t}+q(v)_{x}-S \text { is bounded in } W^{-1, r}(\Omega), \text { for } r>1
$$

That is,

$$
A+R+B+\Sigma \text { is bounded in } W^{-1, r}(\Omega), \quad r>1 .
$$

It follows from (5.31)-(5.32) and Lemma 5.4 that

$$
A+R+B+\Sigma \text { is compact in } H_{\text {loc }}^{-1}(\Omega) \text {. }
$$

That is,

$$
\eta(v)_{t}+q(v)_{x}-S \text { is compact in } H_{\text {loc }}^{-1}(\Omega) .
$$

By (5.28) and (5.34), we have (5.14).

Combining Theorem 4.2 and Theorem 5.5, we have the following framework of the approximate solutions $v_{h}$ defined in Sect. 3 .

Theorem 5.6. Suppose that the initial data $\left(\rho_{0}(x), m_{0}(x)\right)$ and given functions $D(x)$ and $\tau$ satisfy the following conditions:

$$
\begin{gathered}
0 \leqq \rho_{0}(x) \leqq M_{1}, \quad \rho_{0}(x) \not \equiv 0, \quad \text { and }\left|m_{0}(x)\right| \leqq M_{2} \rho_{0}(x), \\
|D(x)| \leqq M_{3}, \quad 0<\tau_{0} \leqq \tau .
\end{gathered}
$$

Then, the approximate solutions $v_{h}$ satisfy the following:

(1) There is a constant $C(T)>0$ such that

$$
0 \leqq \rho_{h}(x, t) \leqq C, \quad\left|m_{h}(x, t)\right| \leqq C \rho_{h}(x, t), \quad(x, t) \in \bar{I}_{T} .
$$

(2) For every domain $\Omega \subset \bar{I}_{T}$ and every weak entropy pair $(\eta, q)$, the sequence of entropy dissipation measures $\eta\left(v_{h}\right)_{t}+q\left(v_{h}\right)_{x}$ is compact in $H_{\mathrm{loc}}^{-1}(\Omega)$. 


\section{Convergence and the Existence Theorem}

In this section, we establish the convergence of a sequence of approximate solutions $v_{h}$ derived by the Godunov scheme and prove the existence of a physical weak solution of the one-dimensional hydrodynamic model (1.1)-(1.3).

For the given function $\tau=\tau(\rho, m)$, we assume that $\tau$ satisfies the uniform Lipschitz condition, namely,

$$
\left|\tau\left(\rho_{2}, m_{2}\right)-\tau\left(\rho_{1}, m_{1}\right)\right| \leqq L\left(\left|\rho_{2}-\rho_{1}\right|+\left|m_{2}-m_{1}\right|\right),
$$

for a constant $L>0$ independent of $(\rho, m)$. Now, we can state and show the main theorem of this paper.

Theorem 6.1. Suppose that the conditions of Theorem 5.6 are satisfied. Then,

(1) For $1<\gamma \leqq \frac{5}{3}$, the sequence of the approximate solutions $v_{h}=\left(\rho_{h}, m_{h}\right)$ has a convergent subsequence, still labeled $v_{h}$, such that

$$
\left(\rho_{h}(x, t), m_{h}(x, t)\right) \rightarrow(\rho(x, t), m(x, t)) \text { a.e. }
$$

and there is a constant $C(T)>0$ such that

$$
0 \leqq \rho(x, t) \leqq C, \quad|m(x, t)| \leqq C \rho(x, t) \text { a.e. }
$$

(2) For $\tau$ satisfying (6.1), the bounded measurable function pair $(\rho(x, t), m(x, t))$ is a physical weak solution of (1.13)-(1.14); i.e., $(\rho, m)$ satisfies $(1.15)-(1.16)$ and (1.18).

Proof. (1) From Theorem 5.6 and the result of [4], we obtain a convergent subsequence, still labeled $v_{h}$, such that

$$
\left(\rho_{h}(x, t), m_{h}(x, t)\right) \rightarrow(\rho(x, t), m(x, t)) \text { a.e. }
$$

Clearly, $0 \leqq \rho(x, t) \leqq C,|m(x, t)| \leqq C \rho(x, t)$ a.e.

(2) (a) For every function $\psi \in C_{0}^{\infty}\left(I_{T}\right)$ satisfying $\psi(x, T)=0$, and $\psi(0, t)=\psi(1, t)=0$, we consider the following integral identity:

$$
\int_{0}^{T} \int_{0}^{1}\left(\rho_{h} \psi_{t}+m_{h} \psi_{x}\right) d x d t+\int_{t=0} \rho_{h} \psi d x=A(\psi)+R(\psi),
$$

where

$$
\begin{aligned}
& A(\psi)=\sum_{i, j} \int\left(\rho_{\bar{h}}^{i}-\rho_{j}^{i}\right) \psi^{i} d x, \\
& R(\psi)=\sum_{i, j}^{t_{i+1}} \int_{t_{2}} \int\left(m_{h}-\underline{m}_{h}\right) \psi_{x} d x d t .
\end{aligned}
$$

We claim that $A(\psi), R(\psi) \rightarrow 0$, as $h \rightarrow 0$. Using Hölders's inequality, (5.1), and the fact that $\rho_{j}^{i}$ is the average value of $\rho_{h}$ on the cell $\left(x_{j-1}, x_{j}\right)$, we have

$$
\begin{aligned}
|A(\psi)| & =\left|\sum_{i, j} \int\left(\rho_{h}^{i}-\rho_{j}^{i}\right)\left(\psi^{i}-\psi_{j}^{i}\right) d x\right| \\
& \leqq\left(\sum_{i, j} \int\left|\psi^{i}-\psi_{j}^{i}\right|^{2} d x\right)^{\frac{1}{2}}\left(\sum_{i, j} \int\left|\rho_{h}^{i}-\rho_{j}^{i}\right|^{2} d x\right)^{\frac{1}{2}} \\
& \leqq\left(\frac{h}{2}\right)^{\frac{1}{2}}\|\psi\|_{C^{1}}\left(\sum_{i, j} \int\left|x-x_{j}\right| d x\right)^{\frac{1}{2}}\left(\sum_{i, j} \int\left|\rho_{\frac{i}{h}}-\rho_{j}^{i}\right|^{2} d x\right)^{\frac{1}{2}} \\
& \leqq C h^{\frac{1}{2}}\|\psi\|_{C^{1}} \rightarrow 0, \text { as } h \rightarrow 0 .
\end{aligned}
$$


It follows from the uniform bound for $v_{h}$ that

$$
\begin{aligned}
R(\psi) & =\left|\sum_{i, j} \int_{t_{i}}^{t_{2}+1} \int V_{2}\left(\underline{v}_{h}\right) \psi_{x}\left(t-t_{i}\right) d x d t\right| \\
& \leqq k\left\|V_{2}\left(\underline{v}_{h}\right)\right\|_{\infty} \int_{0}^{T} \int_{0}^{1}\left|\psi_{x}\right| d x d t \\
& \leqq C h\|\psi\|_{C^{1}} \rightarrow 0, \quad \text { as } h \rightarrow 0 .
\end{aligned}
$$

Then, (6.7)-(6.8) imply that

$$
\lim _{h \rightarrow 0} \int_{0}^{T} \int_{0}^{1}\left(\rho_{h} \psi_{t}+m_{h} \psi_{x}\right) d x d t+\int_{t=0} \rho_{h} \psi d x=0 .
$$

Applying the dominated convergence theorem to (6.9), we have

$$
\int_{0}^{T} \int_{0}^{1}\left(\rho \psi_{t}+m \psi_{x}\right) d x d t+\int_{t=0} \rho_{0}(x) \psi d x=0 .
$$

(b) For every function $\psi \in C_{0}^{1}\left(I_{T}\right)$ satisfying $\psi(0, t)=\psi(1, t)=0$ for $t \geqq 0$ and $\psi(x, T)=0$ for $0 \leqq x \leqq 1$, we consider the following integral identity:

$$
\int_{0}^{T} \int_{0}^{1}\left(m_{h} \psi_{t}+f_{2}\left(v_{h}\right) \psi_{x}+V_{2}\left(v_{h}\right) \psi\right) d x d t+\int_{t=0} m_{h} \psi d x=A(\psi)+R(\psi),
$$

where $f_{2}(v)=\frac{m^{2}}{\rho}+\frac{\rho^{\gamma}}{\gamma}$,

$$
A(\psi)=\sum_{i, j} \int\left(\underline{m}_{h}^{i}-m_{j}^{i}\right) \psi^{i} d x+\sum_{i, j} \iint V_{2}\left(\underline{v}_{h}\right) \psi d x d t
$$

and

$$
\begin{aligned}
R(\psi)= & \sum_{i, j} \iint\left[\left(m_{h}-\underline{m}_{h}\right) \psi_{t}+\left(f_{2}\left(v_{h}\right)-f_{2}\left(\underline{v}_{h}\right)\right) \psi_{x}\right. \\
& \left.+\left(V_{2}\left(v_{h}\right)-V_{2}\left(\underline{v}_{h}\right)\right) \psi\right] d x d t,
\end{aligned}
$$

where $\iint=\int_{t_{\imath}}^{t_{t}+1} \int_{x_{j-1}}^{x_{j}}$.

We estimate $R(\psi)$ first. By (6.1), the uniform bound for $v_{h}$, and the inequality $\left|m_{h}-\underline{m}_{h}\right| \leqq\left|V_{2}\left(\underline{v}_{h}\right)\right| k$, we have

$$
\begin{aligned}
|R(\psi)| & \leqq k \sum_{i, j} \int_{t_{i}}^{t_{i+1}} \int\left[\left|\psi_{t}\right|+\left|\psi_{x}\right|+\left|f_{2}^{\prime}\left(\xi_{j}^{i}\right)\right|+|\psi|\left(\frac{1}{\tau_{0}}+\frac{L\left|\underline{m}_{h}\right|}{\tau_{0}^{2}}\right)\right]\left|V_{2}\left(\underline{v}_{h}\right)\right| d x d t \\
& \leqq C h\|\psi\|_{C^{1}} \rightarrow 0 \quad \text { as } h \rightarrow 0
\end{aligned}
$$

where $\xi_{j}^{i}$ is the mean value of $v_{h}$ on the region $\left(x_{j-1}, x_{j}\right) \times\left(t_{i}, t_{i+1}\right)$.

To estimate $A(\psi)$, we decompose $A(\psi)$ into three parts:

$$
\begin{aligned}
A(\psi)= & \left\{\sum_{i, j} \int\left(\underline{m}_{h}^{i}-m_{j}^{i}\right)\left(\psi^{i}-\psi_{j}^{i}\right) d x\right\} \\
& +\left\{\sum_{i, j} \int_{t_{i}}^{t_{i+1}} \int V_{2}\left(\underline{v}_{h}\right)\left(\psi-\psi_{j}^{i}\right) d x d t\right\} \\
& +\left\{\sum_{i, j} \int_{t_{i}}^{t_{i+1}} \int\left(V_{2}\left(\underline{v}_{h}\right)-V_{2}\left(\underline{v}_{h}^{i}\right)\right) \psi_{j}^{i} d x d t\right\} \\
\equiv & A_{1}(\psi)+A_{2}(\psi)+A_{3}(\psi) .
\end{aligned}
$$


For $A_{1}(\psi)$ and $A_{2}(\psi)$, we have

$$
\begin{aligned}
\left|A_{1}(\psi)\right|= & \left|\sum_{i, j} \int\left(k V_{2}\left(\underline{v}_{h}^{i}\right)+m_{h}^{i}-m_{j}^{i}\right)\left(\psi^{i}-\psi_{j}^{i}\right) d x\right| \\
\leqq & \left(\sum_{i, j} \int\left(\psi^{i}-\psi_{j}^{i}\right)^{2} d x\right)^{\frac{1}{2}}\left[k\left(\sum_{i, j} \int V_{2}^{2}\left(\underline{v}_{h}^{i}\right) d x\right)^{\frac{1}{2}}\right. \\
& \left.+\left(\sum_{i, j} \int\left(m_{\bar{h}}^{i}-m_{j}^{i}\right)^{2} d x\right)^{\frac{1}{2}}\right] \quad \text { by Lemma 5.1) } \\
\leqq & \left(\frac{h}{2}\right)^{\frac{1}{2}}\|\psi\|_{C^{1}}\left(n \sum_{j} \int\left|x-x_{j}\right| d x\right)^{\frac{1}{2}}\left(T\left\|V_{2}^{2}\left(\underline{v}_{h}\right)\right\|_{\infty}+C\right) \\
\leqq & C h^{\frac{1}{2}}\|\psi\|_{C^{1}} \rightarrow 0 \text { as } h \rightarrow 0
\end{aligned}
$$

and

$$
\begin{aligned}
\left|A_{2}(\psi)\right| & \leqq \sum_{i, j} \iint\left|V_{2}\left(\underline{v}_{h}\right)\right|\left(\frac{\left|\psi^{i}-\psi_{j}^{i}\right|}{\left|x-x_{j}\right|} h+\frac{\left|\psi(x, t)-\psi^{i}\right|}{\left|t-t_{i}\right|} k\right) d x d t \\
& \leqq C h\|\psi\|_{C^{1}} \rightarrow 0, \quad \text { as } h \rightarrow 0 .
\end{aligned}
$$

By (6.1) and Lemmas 2.4 and 2.5, we have

$$
\begin{aligned}
& \left|A_{3}(\psi)\right|=\left|\sum_{i, j} \psi_{j}^{i} \iint\left(V_{2}\left(\underline{v}_{h}\right)-V_{2}\left(\underline{v}_{h}^{i}\right)\right) d x d t\right| \\
& \leqq C\|\psi\|_{\infty} \sum_{i, j} \iint\left(\left|\underline{\rho}_{h}-\underline{\rho}_{h} \frac{i}{h}\right|+\left|\underline{m}_{h}-\underline{m} \frac{i}{h}\right|\right) d x d t \\
& \leqq C\|\psi\|_{\infty} \sum_{i, j} \iint\left|\underline{\rho}_{h}-\underline{\rho}_{h}^{i}\right| d x d t \quad \text { (by Lemma 2.4) } \\
& \leqq C\|\psi\|_{\infty} \sum_{i, j} \int_{t_{l}}^{t_{2}+1} \varepsilon h d t \quad \text { (by Lemma 2.5) } \\
& \leqq C \varepsilon\|\psi\|_{\infty},
\end{aligned}
$$

where $\varepsilon>0$ is an arbitrarily small constant.

It follows from (6.14)-(6.18) that

$$
\lim _{h \rightarrow 0} \int_{0}^{T} \int_{0}^{1}\left(m_{h} \psi_{t}+f_{2}\left(v_{h}\right) \psi_{x}+V_{2}\left(v_{h}\right) \psi\right) d x d t+\int_{t=0} m_{h} \psi d x=0 .
$$

Using the dominated convergence theorem, we have

$$
\begin{aligned}
& \int_{0}^{T} \int_{0}^{1}\left(m \psi_{t}+\left(\frac{m^{2}}{\rho}+\frac{\rho^{\gamma}}{\gamma}\right) \psi_{x}\right) d x d t \\
& \left.\quad+\int_{0}^{T} \int_{0}^{1}\left(\rho \int_{0}^{1} G_{x}(\rho-D) d \xi-\frac{m}{\tau}\right) \psi \tau\right) \psi d x d t+\int_{t=0} m_{0}(x) \psi d x=0 .
\end{aligned}
$$

(c) For every weak and convex entropy pair $(\eta, q)$ and every nonnegative smooth function $\widetilde{\psi}$ that has compact support in region $I_{T}$, we consider the 
following integral identity:

$$
\int_{0}^{T} \int_{0}^{1}\left(\eta\left(v_{h}\right) \tilde{\psi}_{t}+q\left(v_{h}\right) \tilde{\psi}_{x}\right) d x d t=A(\tilde{\psi})+R(\tilde{\psi})+\Sigma(\tilde{\psi})+S(\tilde{\psi}),
$$

where $A(\tilde{\psi}), R(\tilde{\psi}), \Sigma(\tilde{\psi})$, and $S(\tilde{\psi})$ are similar to those of (5.15).

Since $(\eta, q)$ is a convex entropy pair and $\widetilde{\psi} \geqq 0$, we have

$$
\begin{gathered}
\sum(\tilde{\psi}) \geqq 0, \\
A(\tilde{\psi})=\sum_{i, j} \tilde{\psi}_{j}^{i} \int\left(v_{\bar{h}}^{i}-v_{j}^{i}\right)^{T} \nabla^{2} \eta\left(\xi_{j}^{i}\right)\left(v_{h}^{i}-v_{j}^{i}\right) d x \\
+\sum_{i, j} \int\left(\eta\left(v_{\bar{h}}^{i}\right)-\eta\left(v_{j}^{i}\right)\right)\left(\tilde{\psi}^{i}-\tilde{\psi}_{j}^{i}\right) d x \\
\geqq \sum_{i, j} \int\left(\eta\left(v_{\bar{h}}^{i}\right)-\eta\left(v_{j}^{i}\right)\right)\left(\tilde{\psi}^{i}-\tilde{\psi}_{j}^{i}\right) d x \\
\geqq-C h^{\alpha-\frac{1}{2}}\|\tilde{\psi}\|_{C_{0}^{\alpha}}, \quad \frac{1}{2}<\alpha<1 .
\end{gathered}
$$

As in (5.27), we have

$$
S(\tilde{\psi}) \geqq-C h\|\tilde{\psi}\|_{H_{0}^{1}}
$$

Using the fact that $\rho_{h}(x, t)=\underline{\rho}(x, t)$ and

$$
m_{h}(x, t)=\underline{m}_{h}(x, t)+V_{2}\left(\underline{v}_{h}(x, t)\right)\left(t_{i}-t_{i-1}\right)
$$

for $t_{i-1} \leqq t<t_{i}$, we obtain

$$
\left|V_{2}\left(v_{h}^{i}\right)-V_{2}\left(\underline{v}_{h}^{i}\right)\right| \leqq C h .
$$

Then,

$$
\begin{aligned}
& R(\tilde{\psi})=-k \sum_{i, j} \int\left(\int_{0}^{1} \eta_{m}\left(v_{\bar{h}}^{\underline{i}}+\theta\left(\underline{v}_{h}^{\underline{i}}-v_{\underline{h}}^{\underline{i}}\right)\right) d \theta\right) V_{2}\left(\underline{v}_{\bar{h}}^{\underline{i}}\right) \tilde{\psi}^{i} d x \\
& =-k \sum_{i, j} \int\left(\int_{0}^{1} \eta_{m}\left(v_{h}^{i}+\theta\left(\underline{v}_{h}^{i}-v^{\frac{i}{h}}\right)\right) d \theta\right)\left(V_{2}\left(\underline{v}_{h}^{\underline{i}}\right)-V_{2}\left(v_{h}^{\underline{i}}\right)\right) \tilde{\psi}^{i} d x \\
& \left.-k \sum_{i, j} \int\left(\int_{0}^{1} \eta_{m}\left(v_{h}^{i}+\theta\left(\underline{v}_{h}^{i}-v \frac{i}{h}\right)\right) d \theta\right) V_{2}\left(v_{h}^{i}\right)\right) d x \tilde{\psi}^{i} \\
& \geqq-C h-\sum_{i, j} k \int_{0}^{1}\left(\int_{0}^{1} \eta_{m}\left(v_{h}^{i}+\theta\left(\underline{v}_{h}^{\underline{i}}-v_{\underline{h}}^{\underline{i}}\right)\right) d \theta\right) V_{2}\left(v_{\underline{h}}^{\underline{i}}\right) \tilde{\psi}^{i} d x .
\end{aligned}
$$

It follows from (6.21)-(6.25) that

$$
\begin{gathered}
\int_{0}^{T} \int_{0}^{1}\left(\eta\left(v_{h}\right) \tilde{\psi}_{t}+q\left(v_{h}\right) \tilde{\psi}_{x}\right) d x d t+\sum_{i=1}^{n} k \int_{0}^{1}\left(\int_{0}^{1} \eta_{m}\left(v_{h}^{i}+\theta\left(\underline{v}_{h}^{i}-v_{h}^{\underline{i}}\right)\right) d \theta\right) V_{2}\left(v_{h}^{\underline{i}}\right) \tilde{\psi}^{i} d x \\
\geqq-C\left(\|\tilde{\psi}\|_{C_{0}^{\alpha}}+h^{\frac{3}{2}-\alpha}\left(1+\|\tilde{\psi}\|_{\boldsymbol{H}_{0}^{1}}\right)\right) h^{\alpha-\frac{1}{2}}, \quad \frac{1}{2}<\alpha<1
\end{gathered}
$$


Letting $h \rightarrow 0$ in (6.26) and using the fact that $v_{h} \rightarrow v$ a.e., we obtain the following entropy condition:

$$
\int_{0}^{T} \int_{0}^{1}\left(\eta(v) \tilde{\psi}_{t}+q(v) \tilde{\psi}_{x}\right) d x d t+\int_{0}^{T} \int_{0}^{1} \eta_{m}(v) V_{2}(v) \tilde{\psi} d x d t \geqq 0 .
$$

This completes the proof of the main result.

Remark. Since $0 \leqq \rho_{h}(x, t) \leqq C,\left|m_{h}(x, t)\right| \leqq C \rho_{h}(x, t)$, and $\left(\rho_{h}(x, t), m_{h}(x, t)\right) \rightarrow$ $(\rho(x, t)), m(x, t))$ a.e., we can define $u$ by $u(x, t)=\frac{m(x, t)}{\rho(x, t)}$ a.e. $(u(x, t)=0$, if $\rho(x, t)=0)$. Then, $(\rho, u)$ satisfies the constraints that $0 \leqq \rho(x, t) \leqq C,|u(x, t)| \leqq C$ a.e. and the following pair of integral identities and entropy condition:

$$
\begin{aligned}
& \int_{0}^{T} \int_{0}^{1}\left(\rho \psi_{t}+\rho u \psi_{x}\right) d x d t+\int_{t=0} \rho_{0} \psi d x=0, \\
& \quad \int_{0}^{T} \int_{0}^{1}\left(\rho u \psi_{t}+\left(\rho u^{2}+p(\rho)\right) \psi_{x}\right) d x d t \\
& \quad+\int_{0}^{T} \int_{0}^{1}\left(\int_{0}^{1} G_{x}(\rho-D) d \xi-\frac{u}{\tau}\right) \rho \psi d x d t+\int_{t=0} \rho_{0} u_{0} \psi d x=0, \\
& \int_{0}^{T} \int_{0}^{1}\left(\eta(\rho, \rho u) \tilde{\psi}_{t}+q(\rho, \rho u) \tilde{\psi}_{x}\right) d x d t \\
& \quad+\int_{0}^{T} \int_{0}^{1} \eta_{\rho u}(\rho, \rho u)\left(\int_{0}^{1} G_{x}(\rho-D) d \xi-\frac{u}{\tau}\right) \rho \tilde{\psi} d x d t \geqq 0,
\end{aligned}
$$

where $\psi$ and $\tilde{\psi}$ are the same as in Definition 1.1 and Theorem 1.1, respectively.

Acknowledgements. The author is grateful to Professors Jim Douglas, Jr., and Peter Markowich for introducing this interesting problem to him. He is also thankful to Professor Gui-Qiang Chen for the helpful discussion with him.

\section{References}

1. Baccarani, G., Wordeman, M.R.: An investigation of steady-state velocity overshoot effects in Si and GaAs devices. Solid State Electron. 28, 404-416 (1985)

2. Bell, T.E.: The quest for ballistic action. IEEE Spectrum 23, 36-38 (1986)

3. Blotekjer, K.: Transport equations for electrons in two-valley semiconductors. IEEE Trans. Electron Devices ED-17, 38-47 (1970)

4. Chen, G.-Q.: Convergence of Lax-Friedrichs scheme for isentropic gas dynamics, III. Acta Math. Sci. 6, 75-120 (1986)

5. Chen, G.-Q.: Limit behavior of approximate solutions to conservation laws. In: Multidimensional Hyperbolic Problems and Computations. Glimm, J., Majda, A.J. (eds.), Berlin Heidelberg, New York: Springer 1991, pp. 38-57

6. Courant, R., Friedrichs, K.O.: Supersonic Flow and Shock-Waves. Interscience, New York: J. Wiley and Sons 1967

7. Degond, P., Markowich, P.A.: On a one-dimensional steady-state hydrodynamic model for semiconductors. Appl. Math. Letters 3(3), 25-29 (1990)

8. Ding, X., Chen, G.-Q., Lou, P.: Convergence of the Lax-Friedrichs scheme for isentropic gas dynamics, I, II. Acta Math. Sci. 5, 415-432, 433-472 (1985) 
9. Ding, X., Chen, G.-Q., Lou, P.: Convergence of the fraction step Lax-Friedrichs scheme and Godunov scheme for the isentropic system of gas dynamics. Commun. Math. Phys. 121, 63-84 (1989)

10. Ding, X., Chen, G.-Q., Lou, P.: A supplement of the paper Convergence of Lax-Friedrichs scheme for isentropic gas dynamics, II-III. Acta Math. Sci. 9, 43-44 (1989)

11. Di Perna, R.J.: Convergence of the viscosity method for isentropic gas dynamics. Commun. Math. Phys. 91, 1-30 (1983)

12. Di Perna, R.J.: Convergence of approximate solutions to conservation laws. Arch. Rat. Mech. Anal. 82, 27-70 (1983)

13. Evans, L.C.: Weak Convergence Methods for Nonlinear Partial Differential Equations. CBMS Lecture Notes of A.M.S., 1990

14. Gamba, I.M.: Stationary transonic solutions for a one-dimensional hydrodynamic model for semiconductors. Commun. in P.D.E. 17, 553-577 (1992)

15. Gardner, C.L.: Numerical simulation of a steady-state electron shock wave in a submicron semiconductor device. IEEE Transactions on Electron Devices 38, 392-398 (1991)

16. Gardner, CL., Jerome, J.M., Rose, Donald J.: Numerical methods for the hydrodynamic device model. IEEE Trans. Computer-Aided Design 8, 501-507 (1989)

17. Godunov, S.: A difference method for numerical calculation of discontinuous solutions of the equations of hydrodynamics. Mat. Sb. 47, 271-360 (1959)

18. Lax, P.D.: Hyperbolic systems of conservation laws and mathematical theory of shock waves. Regional Conference Series in Applied Mathematics, SIAM, Philadelphia 11, (1973)

19. Lax, P.D.: Shock waves and entropy. In Contributions to Nonlinear Functional Analysis. Zarantonello, E. (ed.), New York: Academic Press 1971, pp. 603-604

20. Liu, T.-P.: Initial-boundary value problems for gas dynamics. Arch. Rat. Mech. Anal. 64, 137-168 (1977)

21. Liu, T.-P., Smoller, J.: The vacuum state in isentropic gas dynamics. Adv. Appl. Math. 1, 345-359 (1980)

22. Markowich, P.A., Ringhofer, C., Schmeiser, C.: Semiconductor Equations. Berlin, Heidelberg, New York. Springer 1990

23. Murat, F.: Compacité par compensation. Ann. Scuola Norm. Sup. Pisa Sci. Fis. 5, 489-507 (1978)

24. Nishida, T., Smoller, J.: Mixed problem for nonlinear conservation laws. J. Diff. Eqs. 23, 244-269 (1977)

25. Rudan, M., Odeh, F., White, J.: Numerical solution of the hydrodynamic model for onedimensional semiconductor device. COMPEL 6, 151-170 (1987)

26. Smoller, J.: Shock Waves and Reaction - Diffusion Equations. Berlin, Heidelberg, New York: Springer 1983

27. Takeno, S.: Initial boundary value problems for isentropic gas dynamics. Proceedings of the Royal Society of Edinburgh 120 A, 1-23 (1992)

28. Tartar, L.: Compensated compactness and application to partial differential equations. In: Research Notes in Math., Nonlinear Analysis and Mechanics. Knops, R.J. (ed.), Vol. 4, New York: Pitman Press 1979

29. Thomann, E., Odeh, F.: Remarks on the well-posedness of the hydrodynamic model for semiconductor devices. In: Proceedings of The Sixth International Nasecde Conference. Miller, J.J.H. (ed.), Boole Press Ltd., 1989

30. Wang, J.H.: A nonhomogeneous system of equations of nonisentropic gas dynamics. Carasso, C., Raviart, P.-A., Serre, D. (eds.) Nonlinear Hyperbolic Problems. Berlin, Heidelberg, New York: Springer 1986

31. Zhang, B.: On a local existence theorem for a one-dimensional hydrodynamic model of semiconductor devices. Technical Report No. 195, Center for Applied Mathematics, Purdue University 1992. To appear in SIAM J. Math. Anal.

32. Zhang, B.: On the viscosity solution for a one-dimensional hyrdodynamic model for semiconductor devices. Technical Report No. 196, Center for Applied Mathematics, Purdue University, 1992, submitted to Math. Meth. Appl. Sci. 